\title{
Higher integrability for weak solutions to a degenerate parabolic system with singular coefficients
}

Yan Dong ${ }^{1}$, Guangwei Du² ${ }^{2 *}$ and Kelei Zhang ${ }^{3}$

\section{"Correspondence:}

guangwei87@mail.nwpu.edu.cn

${ }^{2}$ School of Mathematical Sciences,

Qufu Normal University, Qufu, China

Full list of author information is

available at the end of the article

\section{Abstract}

In this paper, we study the degenerate parabolic system

$$
u_{t}^{i}+X_{\alpha}^{*}\left(a_{i j}^{\alpha \beta}(z) X_{\beta} u^{j}\right)=g_{i}(z, u, X u)+X_{\alpha}^{*} f_{i}^{\alpha}(z, u, X u),
$$

where $X=\left\{X_{1}, \ldots, X_{m}\right\}$ is a system of smooth real vector fields satisfying Hörmander's condition and the coefficients $a_{i j}^{\alpha \beta}$ are measurable functions and their

skew-symmetric part can be unbounded. After proving the $L^{2}$ estimates for the weak solutions, the higher integrability is proved by establishing a reverse Hölder inequality for weak solutions.

MSC: Primary 35K65; secondary 35K40; 35B65

Keywords: Degenerate parabolic system; Hörmander's vector fields; $L^{2}$ estimates; Higher integrability

\section{Introduction}

Let $\left\{X_{1}, \ldots, X_{q}\right\}$ be a system of smooth real vector fields in a neighborhood $\widetilde{\Omega}$ of some bounded domain $\Omega \subset \mathbb{R}^{n}(n \geq q)$, satisfying Hörmander's rank condition up to the order $s$ and free up to the order $s$. The main purpose of this paper is to study higher integrability for weak solutions to nondiagonal quasilinear degenerate parabolic system

$$
u_{t}^{i}+X_{\alpha}^{*}\left(a_{i j}^{\alpha \beta}(z) X_{\beta} u^{j}\right)=g_{i}(z, u, X u)+X_{\alpha}^{*} f_{i}^{\alpha}(z, u, X u),
$$

where $i, j=1,2, \ldots, N ; \alpha, \beta=1,2, \ldots, q ; z=(x, t) \in Q_{T}=\Omega \times(0, T) ; X_{\alpha}^{*}=-X_{\alpha}+c_{\alpha}\left(c_{\alpha}=\right.$ $\left.\sum_{k=1}^{n} b_{\alpha k}(x) \frac{\partial}{\partial x_{k}} \in C^{\infty}\right)$ is the transposed vector field of $X_{\alpha}$. The assumptions on functions $g_{i}, f_{i}^{\alpha}$ and the coefficients will be specified later.

A function $u \in W_{2}^{1,1}\left(Q_{T}, \mathbb{R}^{N}\right)$ is called a weak solution to (1.1) if

$$
\iint_{Q_{T}}\left[u_{t}^{i} \psi^{i}+a_{i j}^{\alpha \beta} X_{\alpha} \psi^{i} X_{\beta} u^{j}\right] d z=\iint_{Q_{T}}\left[g_{i} \psi^{i}+f_{i}^{\alpha} X_{\alpha} \psi^{i}\right] d z,
$$

for all $\psi \in C_{0}^{\infty}\left(Q_{T}, \mathbb{R}^{N}\right)$.

\section{Springer}

(c) The Author(s) 2019. This article is distributed under the terms of the Creative Commons Attribution 4.0 International License (http://creativecommons.org/licenses/by/4.0/), which permits unrestricted use, distribution, and reproduction in any medium, provided you give appropriate credit to the original author(s) and the source, provide a link to the Creative Commons license, and indicate if changes were made. 
In the Euclidean space, regularity to elliptic and parabolic equations and systems has been studied by many authors (see [1-8] and the references therein). Giaquinta in [5] proved the reverse Hölder estimates for weak solutions to diagonal elliptic systems with Hölder continuous coefficients and obtained the higher integrability of weak solutions. Giaquinta and Struwe in [2] treated partial regularity for weak solutions to diagonal quasilinear parabolic systems with the natural growth conditions and got Hölder continuity. Wiegner in [9] derived Hölder continuity of weak solutions to nondiagonal elliptic systems with VMO coefficients and natural growth conditions. Recently, Földes and Phan [10] got the higher integrability for gradients of weak solutions to a linear elliptic equation having the skew-symmetric part of coefficients unbounded.

Based on Hömander's fundamental work [11], there has been tremendous work on degenerate PDEs arising from non-commuting vector fields; see, for example, [12-21]. Di Fazio and Fanciullo in [14] obtained gradient estimates for weak solutions to linear diagonal elliptic systems with bounded VMO coefficients. Dong and Niu [17] got the higher $L^{p}$ estimates for the gradient of weak solutions to nondiagonal quasilinear degenerate elliptic systems. In [16, 18], Dong and her collaborators studied Morrey and Hölder regularity for weak solutions to diagonal and nondiagonal parabolic systems with bounded VMO coefficients.

However, as far as we know, there is no relevant research about quasilinear degenerate parabolic systems with skew-symmetric coefficients. In this paper, we try to generalize the results in [10] to quasilinear degenerate parabolic systems constructed by Hörmander's vector fields. The aim of this paper is to get the higher integrability for weak solutions to (1.1). In order to state our results, we make the following hypotheses:

(H1) The coefficients $a_{i j}^{\alpha \beta}(z)=A_{i j}^{\alpha \beta}(z)+B_{i j}^{\alpha \beta}(z)$, where $A_{i j}^{\alpha \beta}$ are symmetric $\left(A_{i j}^{\alpha \beta}=A_{j i}^{\alpha \beta}\right)$, bounded, and satisfy the uniform ellipticity condition that for some $\Lambda>0$,

$$
A_{i j}^{\alpha \beta}(z) \xi_{i}^{\alpha} \xi_{j}^{\beta} \geq \Lambda|\xi|^{2}, \quad\left|A_{i j}^{\alpha \beta}(z)\right| \leq \Lambda^{-1}, \quad \text { a.e. } z \in Q_{T}, \forall \xi \in \mathbb{R}^{q N}
$$

$B_{i j}^{\alpha \beta}(z)$ are skew-symmetric $\left(B_{i j}^{\alpha \beta}=-B_{j i}^{\alpha \beta}\right)$ and belong to BMO space (therefore they can be unbounded).

(H2) For any $(z, u, \xi) \in Q_{T} \times \mathbb{R}^{N} \times \mathbb{R}^{q N}$,

$$
\begin{aligned}
& \left|g_{i}(z, u, \xi)\right| \leq g^{i}(z)+L|\xi|^{\gamma_{0}} \\
& \left|f_{i}^{\alpha}(z, u, \xi)\right| \leq g_{i}^{\alpha}(z)+L|\xi|
\end{aligned}
$$

where $1 \leq \gamma_{0}<1 / q_{0}, q_{0}=\frac{Q+2}{Q+4}, L$ is a positive constant satisfying $L<\Lambda$, and

$$
g^{i}(z) \in L^{p q_{0}}\left(Q_{T}\right), \quad g_{i}^{\alpha} \in L^{p}\left(Q_{T}\right), \quad p \geq 2 .
$$

Here $Q$ is the homogeneous dimension relative to $\Omega$, and in the sequel we set $\tilde{g}=\left(g^{i}\right)$, $\tilde{\tilde{g}}=\left(g_{i}^{\alpha}\right), \tilde{q}=2 q_{0}$.

Now we state our main result.

Theorem 1.1 Suppose that (H1) and (H2) hold. Let $u \in W_{2}^{1,1}\left(Q_{T}, \mathbb{R}^{N}\right)$ be a weak solutions to (1.1), then there exists a constant $\varepsilon_{0}>$ such that for any $p \in\left[2,2+\tilde{q} \varepsilon_{0}\right)$, we have $X u \in$ 


$$
\begin{aligned}
& L_{\mathrm{loc}}^{p}\left(Q_{T}, \mathbb{R}^{N}\right), \text { and for every } Q_{T}^{\prime} \subset \subset Q_{T} \text {, there exists a constant } C>0 \text { such that } \\
& \qquad\|X u\|_{L^{p}\left(Q_{T}^{\prime}\right)} \leq C\left(\|\tilde{g}\|_{L^{p q_{0}}\left(Q_{T}\right)}^{q_{0}}+\|\tilde{\tilde{g}}\|_{L^{p}\left(Q_{T}\right)}\right) .
\end{aligned}
$$

The main difficulty in the proof is establishing the reverse Hölder inequality for gradients of weak solutions. We first establish the $L^{2}$ estimates of weak solutions by constructing suitable test functions. Then the reverse Hölder inequality of gradients is obtained by the $L^{2}$ estimates and the Gehring lemma on a metric measure space.

The paper is organized as follows. In Sect. 2, we introduce some concepts and results related to Hörmander's vector fields that will be used in our proof. Section 3 is devoted to establishing the reverse Hölder inequality for gradients of weak solutions to (1.1) and giving the proof of Theorem 1.1.

\section{Preliminaries}

Let

$$
X_{\alpha}=\sum_{k=1}^{n} b_{\alpha k} \frac{\partial}{\partial x_{k}}, \quad b_{\alpha k} \in C^{\infty}, \alpha=1,2, \ldots, q
$$

be a family of vector fields in a neighborhood $\widetilde{\Omega}$ of some bounded domain $\Omega \subset \mathbb{R}^{n}$. For a multiindex $\alpha=\left(i_{1}, \ldots, i_{k}\right)$, denote by $X_{\beta}=\left[X_{i_{1}},\left[X_{i_{2}}, \ldots,\left[X_{i_{k-1}}, X_{i_{k}}\right]\right] \ldots\right]$ the commutator of vector fields $X_{1}, \ldots, X_{q}$ with length $k=|\beta|$. We say that the vector fields $X_{1}, \ldots, X_{q}$ satisfy Hörmander's condition up to the order $s$ (see [11]) provided there exists $s>0$ such that $\left\{X_{\beta}\right\}_{|\beta| \leq s}$ span the tangent space at each point in $\mathbb{R}^{n}$.

We denote by $X u=\left(X_{1} u, \ldots, X_{q} u\right)$ the gradient of $u$ with respect to the system $X=$ $\left\{X_{1}, \ldots, X_{q}\right\}$ and hence

$$
|X u(x)|=\left(\sum_{\alpha=1}^{q}\left|X_{\alpha} u(x)\right|^{2}\right)^{\frac{1}{2}}
$$

An absolutely continuous curve $\gamma:[a, b] \rightarrow \tilde{\Omega}$ is said to be admissible for the family $X$, if there exist functions $c_{\alpha}(t), a \leq t \leq b$, satisfying

$$
\sum_{\alpha=1}^{q} c_{\alpha}(t)^{2} \leq 1 \quad \text { and } \quad \gamma^{\prime}(t)=\sum_{\alpha=1}^{q} c_{\alpha}(t) X_{\alpha}(\gamma(t)), \quad \text { a.e. } t \in[a, b] \text {. }
$$

The Carnot-Carathéodory distance induced by $X$ is defined by

$$
d_{X}(x, y)=\inf \{T>0 \text { : there is an admissible curve } \gamma, \gamma(0)=x, \gamma(T)=y\} .
$$

Then $d_{X}$ is a local metric on $\tilde{\Omega}$. The metric ball is denoted by

$$
B_{R}(x)=B(x, R)=\left\{y \in \Omega: d_{X}(x, y)<R\right\} .
$$

If one does not need to consider the center of the ball, then we also write $B_{R}$ instead of $B(x, R)$. 
It is well known that the doubling property for metric balls holds true (see $[22,23])$ : there exist positive constants $R_{d}>0$ and $C_{d} \geq 1$ such that for any $x \in \Omega$ and $0<2 R \leq R_{d}$,

$$
|B(x, 2 R)| \leq C_{d}|B(x, R)| .
$$

Here, $|B(x, R)|$ denotes the Lebesgue measure of $B(x, R)$. The number $Q=\log _{2} C_{d}$ is called the homogeneous dimension relative to $\Omega$. Clearly, $Q \geq n$. From the doubling property, we can see that

$$
\left|B_{t R}\right| \geq C t^{Q}\left|B_{R}\right|, \quad \forall R \leq R_{d}, t \in(0,1)
$$

where $C=C_{d}^{-2}$. In particular, if the vector fields $X_{1}, \ldots, X_{q}$ are free up to the order $s$, there exist two positive constants $C_{1}$ and $C_{2}$ such that ([24])

$$
C_{1} R^{Q} \leq|B(x, R)| \leq C_{2} R^{Q}
$$

For $z_{0}=\left(x_{0}, t_{0}\right) \in Q_{T} \subset \mathbb{R}^{n+1}$, the parabolic cylinder with vertex at $z_{0}$ is defined by

$$
Q_{R}\left(z_{0}\right)=B_{R}\left(x_{0}\right) \times\left(t_{0}-\frac{R^{2}}{2}, t_{0}+\frac{R^{2}}{2}\right] .
$$

Let $I_{R}\left(t_{0}\right)=\left(t_{0}-\frac{R^{2}}{2}, t_{0}+\frac{R^{2}}{2}\right]$, and the parabolic boundary of $Q_{R}\left(z_{0}\right)$ be denoted by

$$
\partial_{p} Q_{R}\left(z_{0}\right)=\left(\partial B_{R}\left(x_{0}\right) \times\left(t_{0}-\frac{R^{2}}{2}, t_{0}+\frac{R^{2}}{2}\right]\right) \cup\left(B_{R}\left(x_{0}\right) \times\left\{t_{0}-\frac{R^{2}}{2}\right\}\right) .
$$

For any $(x, t),(y, s) \in Q_{T}$, the parabolic distance in $Q_{T}$ is defined by

$$
d_{p}((x, t),(y, s))=\sqrt{d_{X}(x, y)^{2}+|t-s|},
$$

and the parabolic ball is defined by

$$
B_{p}\left(z_{0}, R\right)=\left\{(x, t) \in Q_{T}: d_{p}\left(\left(x_{0}, t_{0}\right),(x, t)\right)<R\right\} .
$$

To simplify the notations, in the sequel, $Q_{R}\left(z_{0}\right), B_{R}\left(x_{0}\right)$, and $I_{R}\left(t_{0}\right)$ are written as $Q_{R}, B_{R}$, and $I_{R}$, respectively. Furthermore, if $E$ is a Lebesgue measurable set with Lebesgue measure $|E|$, we set $u_{E}=f_{E} u d x$ to be the integral average of $u$ on $E$.

We define the parabolic Sobolev space by

$$
W_{p}^{1,1}\left(Q_{T}\right)=\left\{u \in L^{p}\left(Q_{T}\right): X_{\alpha} u, \partial_{t} u \in L^{p}\left(Q_{T}\right), \alpha=1,2, \ldots, q\right\}
$$

with the norm

$$
\|u\|_{W_{p}^{1,1}\left(Q_{T}\right)}=\|u\|_{L^{p}\left(Q_{T}\right)}+\left\|\partial_{t} u\right\|_{L^{p}\left(Q_{T}\right)}+\sum_{\alpha=1}^{q}\left\|X_{\alpha} u\right\|_{L^{p}\left(Q_{T}\right)} .
$$


For any $f \in L_{\text {loc }}^{1}\left(Q_{T}\right)$, if

$$
\begin{aligned}
\|f\|_{\mathrm{BMO}} & =\sup _{z_{0} \in Q_{T}, \rho>0} \frac{1}{\left|Q_{T} \cap Q_{\rho}\left(z_{0}\right)\right|} \iint_{Q_{T} \cap Q_{\rho}\left(z_{0}\right)}\left|f-f_{\mathrm{Q}_{T} \cap Q_{\rho}\left(z_{0}\right)}\right| d z \\
& <\infty
\end{aligned}
$$

we say that $f \in \mathrm{BMO}\left(Q_{T}\right)$ (i.e., $f$ has bounded mean oscillation).

Lemma 2.1 (Sobolev inequality, see [12, 23]) For every compact set $K \subset \Omega$, there exist constants $C>0$ and $\bar{R}>0$ such that for any metric ball $B=B\left(x_{0}, R\right)$ with $x_{0} \in K$ and $0<$ $R \leq \bar{R}$, it holds that for any $f \in C^{\infty}\left(\overline{B_{R}}\right)$,

$$
\left(f_{B_{R}}\left|f-f_{R}\right|^{\kappa p} d x\right)^{\frac{1}{\kappa p}} \leq C R\left(f_{B_{R}}|X f|^{p} d x\right)^{\frac{1}{p}}
$$

where $f_{R}=f_{B_{R}} f d x$ is the integral average of $f$ on $B_{R}$, and $1 \leq \kappa \leq Q /(Q-p)$, if $1 \leq p<Q$; $1 \leq \kappa<\infty$, if $p \geq Q$. Moreover,

$$
\left(f_{B_{R}}|f|^{\kappa p} d x\right)^{\frac{1}{\kappa p}} \leq C R\left(f_{B_{R}}|X f|^{p} d x\right)^{\frac{1}{p}}
$$

whenever $f \in C_{0}^{\infty}\left(\overline{B_{R}}\right)$.

Lemma 2.2 (Iterative lemma, see [25]) Let $\varphi(t)$ be a bounded nonnegative function on $\left[T_{0}, T_{1}\right]$, where $T_{1}>T_{0} \geq 0$. Suppose that for any $t$ and $s, T_{0} \leq t<s \leq T_{1}, \varphi(t)$ satisfies

$$
\varphi(t) \leq \theta \varphi(s)+\frac{A}{(s-t)^{\alpha}}+B
$$

where $\theta, A, B$, and $\alpha$ are nonnegative constants with $\theta<1$. Then for any $T_{0} \leq \rho<R \leq T_{1}$, one has

$$
\varphi(\rho) \leq c\left[\frac{A}{(R-\rho)^{\alpha}}+B\right]
$$

where $c$ depends only on $\alpha$ and $\theta$.

The following Gehring lemma on the metric measure space $(Y, d, \mu)(d$ is a metric and $\mu$ is a doubling measure) can be found in $[13,26]$.

Lemma 2.3 Let $q \in\left[q_{0}, 2 Q\right]$, where $q_{0}>1$ is fixed. Assume that functions $f, g$ are nonnegative and $g \in L_{\mathrm{loc}}^{q}(Y, \mu), f \in L_{\mathrm{loc}}^{r_{0}}(Y, \mu)$, for some $r_{0}>q$. If there exist constants $b>1$ and $\theta$ such that for every ball $B \subset \sigma B \subset Y$ the following inequality holds:

$$
f_{B} g^{q} d \mu \leq b\left[\left(f_{\sigma B} g d \mu\right)^{q}+f_{\sigma B} f^{q} d \mu\right]+\theta f_{\sigma B} g^{q} d \mu
$$


then there exist nonnegative constants $\theta_{0}=\theta_{0}\left(q_{0}, Q, C_{d}, \sigma\right)$ and $\varepsilon_{0}=\varepsilon_{0}\left(b, q_{0}, Q, C_{d}, \sigma\right)$ such that if $0<\theta<\theta_{0}$ then $g \in L_{\mathrm{loc}}^{p}(Y, \mu)$ for $p \in\left[q, q+\varepsilon_{0}\right)$ and moreover

$$
\left(f_{B} g^{p} d \mu\right)^{\frac{1}{p}} \leq C\left[\left(f_{\sigma B} g^{q} d \mu\right)^{\frac{1}{q}}+\left(f_{\sigma B} f^{p} d \mu\right)^{\frac{1}{p}}\right]
$$

for some positive constant $C=C\left(q_{0}, Q, C_{d}, \sigma\right)$.

\section{Higher integrability}

We first introduce two cutoff functions $\xi(x)$ and $\eta(t)$ (see to [4]) such that for any $0<\rho<$ $R, B_{\rho} \subset B_{R} \subset \Omega$,

$$
\begin{aligned}
& \xi(x) \in C_{0}^{\infty}\left(B_{R}\right), \quad 0 \leq \xi \leq 1, \quad|X \xi| \leq \frac{C}{R-\rho} \quad \text { and } \quad \xi=1 \text { in } B_{\rho} ; \\
& \eta(t)= \begin{cases}\frac{2 t-2\left(t_{0}-\frac{R^{2}}{2}\right)}{R^{2}-\rho^{2}}, & t \in\left(t_{0}-\frac{R^{2}}{2}, t_{0}-\frac{\rho^{2}}{2}\right), \\
1, & t \in\left[t_{0}-\frac{\rho^{2}}{2}, t_{0}+\frac{R^{2}}{2}\right] .\end{cases}
\end{aligned}
$$

Setting $N_{1}=f_{B_{R}} \xi^{2}(x) d x$, we denote the average of $u(x, t)$ on $B_{R}$ by

$$
\bar{u}(t)=\left(\int_{B_{R}} \xi^{2} d x\right)^{-1} \int_{B_{R}} u \xi^{2} d x=\frac{1}{N_{1}\left|B_{R}\right|} \int_{B_{R}} u \xi^{2} d x
$$

Lemma 3.1 Let $u \in W_{2}^{1,1}\left(\Omega_{T}, \mathbb{R}^{N}\right)$ be a weak solution to (1.1). Then for any $Q_{R} \subset \subset \Omega_{T}$, we have

$$
\int_{B_{R}}|u-\bar{u}(t)|^{2} d x+\iint_{Q_{R}}|X u|^{2} d z \leq c \iint_{Q_{R}}|\tilde{g}|^{\tilde{q}} d z+c \iint_{Q_{R}}|\tilde{\tilde{g}}|^{2} d z .
$$

Proof Multiplying both sides of (1.1) by the test function $u-\bar{u}(t)$ and integrating on $Q_{R}$, we get

$$
\iint_{Q_{R}}\left[u_{t}^{i}+X_{\alpha}{ }^{*}\left(a_{i j}^{\alpha \beta} X_{\beta} u^{j}\right)\right]\left(u^{i}-\bar{u}(t)\right) d z=\iint_{Q_{R}}\left[g_{i}+X_{\alpha}{ }^{*} f_{i}^{\alpha}\right]\left(u^{i}-\bar{u}(t)\right) d z .
$$

So we have

$$
\iint_{Q_{R}}\left[u_{t}^{i}\left(u^{i}-\bar{u}(t)\right)+a_{i j}^{\alpha \beta} X_{\alpha} u^{i} X_{\beta} u^{j}\right] d z=\iint_{Q_{R}}\left[g_{i}\left(u^{i}-\bar{u}(t)\right)+f_{i}^{\alpha} X_{\alpha} u^{i}\right] d z .
$$

By $(\mathrm{H} 1)$, the above can be written as

$$
\begin{aligned}
& \iint_{Q_{R}}\left(\frac{1}{2}\left|u^{i}-\bar{u}(t)\right|^{2}\right)_{t} d z+\iint_{Q_{R}} A_{i j}^{\alpha \beta} X_{\alpha} u^{i} X_{\beta} u^{j} d z \\
& \quad=-\iint_{Q_{R}} B_{i j}^{\alpha \beta} X_{\alpha} u^{i} X_{\beta} u^{j} d z+\iint_{Q_{R}}\left[g_{i}\left(u^{i}-\bar{u}(t)\right)+f_{i}^{\alpha} X_{\alpha} u^{i}\right] d z .
\end{aligned}
$$

Due to the skew-symmetry of $B_{i j}^{\alpha \beta}$,

$$
\iint_{Q_{R}} B_{i j}^{\alpha \beta} X_{\alpha} u^{i} X_{\beta} u^{j} d z=0
$$


By (H2), Hölder's, Sobolev's, and Young's inequalities, we have

$$
\begin{aligned}
& \iint_{Q_{R}} g_{i}\left(u^{i}-\bar{u}(t)\right) d z \\
& \leq \iint_{Q_{R}}\left(g^{i}(z)+L|X u|^{\gamma_{0}}\right)\left(u^{i}-\bar{u}(t)\right) d z \\
& \leq \int_{I_{R}}\left[\left(\int_{B_{R}}|\tilde{g}|^{\tilde{q}} d x\right)^{\frac{1}{\tilde{q}}}\left(\int_{B_{R}}|u-\bar{u}(t)|^{\frac{2(Q+2)}{Q}} d x\right)^{\frac{Q}{2(Q+2)}}\right] d t \\
& +L \int_{I_{R}}\left[\left(\int_{B_{R}}|X u|^{2} d x\right)^{\frac{\gamma_{0}}{2}}\left(\int_{B_{R}}|u-\bar{u}(t)|^{\frac{2}{2-\gamma_{0}}} d x\right)^{\frac{2-\gamma_{0}}{2}}\right] d t \\
& \leq \int_{I_{R}}\left[\left(\int_{B_{R}}|\tilde{g}|^{\tilde{q}} d x\right)^{\frac{1}{\tilde{q}}} c R^{\frac{2}{Q+2}}\left(\int_{B_{R}}|X u|^{2} d x\right)^{\frac{1}{2}}\right] d t \\
& +\int_{I_{R}}\left[\left(\int_{B_{R}}|X u|^{2} d x\right)^{\frac{\gamma_{0}}{2}} c R^{\frac{Q+2-Q \gamma_{0}}{2}}\left(\int_{B_{R}}|X u|^{2} d x\right)^{\frac{1}{2}}\right] d t \\
& \leq c_{\varepsilon} \iint_{Q_{R}}|\tilde{g}|^{\tilde{q}} d z+\varepsilon R^{\frac{4}{Q}} \int_{I_{R}}\left(\int_{B_{R}}|X u|^{2} d x\right)^{\frac{Q+2}{Q}} d t \\
& +c R^{\frac{Q+2-Q \gamma_{0}}{2}} \sup _{I_{R}}\left(\int_{B_{R}}|X u|^{2} d x\right)^{\frac{\gamma_{0}-1}{2}} \iint_{Q_{R}}|X u|^{2} d z \\
& \leq c_{\varepsilon} \iint_{Q_{R}}|\tilde{g}|^{\tilde{q}} d z+\varepsilon R^{\frac{4}{Q}} \sup _{I_{R}}\left(\int_{B_{R}}|X u|^{2} d x\right)^{\frac{2}{Q}} \iint_{Q_{R}}|X u|^{2} d z \\
& +c R^{\frac{Q+2-Q \gamma_{0}}{2}} \sup _{I_{R}}\left(\int_{B_{R}}|X u|^{2} d x\right)^{\frac{\gamma_{0}-1}{2}} \iint_{Q_{R}}|X u|^{2} d z,
\end{aligned}
$$

and

$$
\begin{aligned}
\iint_{Q_{R}} f_{i}^{\alpha} X_{\alpha} u^{i} d z & \leq \iint_{Q_{R}}\left|g_{i}^{\alpha}(z)\right||X u| d z+L \iint_{Q_{R}}|X u|^{2} d z \\
& \leq c_{\varepsilon} \iint_{Q_{R}}|\tilde{\tilde{g}}|^{2} d z+(\varepsilon+L) \iint_{Q_{R}}|X u|^{2} d z .
\end{aligned}
$$

Inserting (3.4), (3.5), and (3.6) into (3.3), and by (H1), we get

$$
\begin{aligned}
& \int_{B_{R}} \frac{1}{2}|u-\bar{u}(t)|^{2} d x+\Lambda \iint_{Q_{R}}|X u|^{2} d z \\
& \quad \leq c_{\varepsilon} \iint_{Q_{R}}|\tilde{g}|^{\tilde{q}} d z+c_{\varepsilon} \iint_{Q_{R}}|\tilde{\tilde{g}}|^{2} d z+\theta \iint_{Q_{R}}|X u|^{2} d z,
\end{aligned}
$$

where $\theta=\varepsilon R^{\frac{4}{Q}} \sup _{I_{R}}\left(\int_{B_{R}}|X u|^{2} d x\right)^{\frac{2}{Q}}+c R^{\frac{Q+2-Q \gamma_{0}}{2}} \sup _{I_{R}}\left(\int_{B_{R}}|X u|^{2} d x\right)^{\frac{\gamma_{0}-1}{2}}+\varepsilon+L$. Because $L<\Lambda$, by choosing $\varepsilon, R$ small enough we can get that $\theta<\Lambda$. So using Lemma 2.2, we complete the proof. 
Lemma 3.2 Let $u \in W_{2}^{1,1}\left(\Omega_{T}, \mathbb{R}^{N}\right)$ be a weak solution of (1.1). Then for any $0<\rho<R$, $Q_{R} \subset \subset \Omega_{T}$, we have

$$
\begin{aligned}
& \sup _{I_{\rho}} \int_{B_{\rho}}|u-\bar{u}(t)|^{2} d x+\iint_{Q_{\rho}}|X u|^{2} d z \\
& \quad \leq \frac{c}{(R-\rho)^{2}} \iint_{Q_{R}}|u-\bar{u}(t)|^{2} d z+c\left(\frac{R^{3}}{(R-\rho)^{2}}+1\right) \iint_{Q_{R}}\left(|\tilde{g}|^{\tilde{q}}+|\tilde{\tilde{g}}|^{2}\right) d z .
\end{aligned}
$$

Proof Let $B_{\rho} \subset B_{R} \subset \Omega$. Multiplying both sides of (1.1) by the test function $(u-\bar{u}(t)) \times$ $\xi^{2}(x) \eta(t)$ and integrating on $Q_{R}^{\prime}=B_{R}\left(x_{0}\right) \times\left(t_{0}-\frac{R^{2}}{2}, s\right]\left(s \leq t_{0}+\frac{R^{2}}{2}\right)$, we get

$$
\begin{gathered}
\iint_{Q^{\prime} R}\left[u_{t}^{i}+X_{\alpha}{ }^{*}\left(a_{i j}^{\alpha \beta} X_{\beta} u^{j}\right)\right]\left(u^{i}-\bar{u}(t)\right) \xi^{2} \eta d z \\
=\iint_{Q^{\prime} R}\left[g_{i}+X_{\alpha}{ }^{*} f_{i}^{\alpha}\right]\left(u^{i}-\bar{u}(t)\right) \xi^{2} \eta d z .
\end{gathered}
$$

By (H1), one has

$$
\begin{aligned}
& \iint_{Q^{\prime}{ }_{R}}\left[u_{t}^{i}+X_{\alpha}^{*}\left(a_{i j}^{\alpha \beta} X_{\beta} u^{j}\right)\right]\left(u^{i}-\bar{u}(t)\right) \xi^{2} \eta d z \\
& =\iint_{Q^{\prime} R}\left[u_{t}^{i}\left(u^{i}-\bar{u}(t)\right) \xi^{2} \eta+a_{i j}^{\alpha \beta} X_{\beta} u^{j} X_{\alpha}\left(\left(u^{i}-\bar{u}(t)\right) \xi^{2} \eta\right)\right] d z \\
& =\iint_{Q^{\prime} R_{R}}\left[u_{t}^{i}\left(u^{i}-\bar{u}(t)\right) \xi^{2} \eta+a_{i j}^{\alpha \beta} \xi^{2} \eta X_{\alpha} u^{i} X_{\beta} u^{j}+2 a_{i j}^{\alpha \beta}\left(u^{i}-\bar{u}(t)\right) \xi \eta X_{\alpha} \xi X_{\beta} u^{j}\right] d z \\
& =\iint_{Q^{\prime}{ }_{R}}\left[\left(\frac{1}{2}\left|u^{i}-\bar{u}(t)\right|^{2} \eta\right) \xi_{t}^{2}-\frac{1}{2}\left|u^{i}-\bar{u}(t)\right|^{2} \xi^{2} \eta_{t}+A_{i j}^{\alpha \beta} \xi^{2} \eta X_{\alpha} u^{i} X_{\beta} u^{j}\right] d z \\
& \quad+\iint_{Q_{\prime_{R}}} B_{i j}^{\alpha \beta} \xi^{2} \eta X_{\alpha} u^{i} X_{\beta} u^{j}+2 a_{i j}^{\alpha \beta}\left(u^{i}-\bar{u}(t)\right) \xi \eta X_{\alpha} \xi X_{\beta} u^{j} d z,
\end{aligned}
$$

and

$$
\begin{aligned}
& \iint_{Q^{\prime} R}\left[g_{i}+X_{\alpha}{ }^{*} f_{i}^{\alpha}\right]\left(u^{i}-\bar{u}(t)\right) \xi^{2} \eta d z \\
& \quad=\iint_{Q_{R}^{\prime}}\left[g_{i}\left(u^{i}-\bar{u}(t)\right) \xi^{2} \eta+f_{i}^{\alpha} X_{\alpha}\left(\left(u^{i}-\bar{u}(t)\right) \xi^{2} \eta\right)\right] d z \\
& \quad=\iint_{Q_{R}^{\prime}}\left[g_{i}\left(u^{i}-\bar{u}(t)\right) \xi^{2} \eta+f_{i}^{\alpha} \xi^{2} \eta X_{\alpha} u^{i}+2 \xi \eta\left(u^{i}-\bar{u}(t)\right) f_{i}^{\alpha} X_{\alpha} \xi\right] d z .
\end{aligned}
$$

By the above, (3.8) can be written as

$$
\begin{aligned}
& \iint_{Q^{\prime} R}\left(\frac{1}{2}\left|u^{i}-\bar{u}(t)\right|^{2} \eta\right)_{t} \xi^{2} d z+\iint_{Q^{\prime} R} A_{i j}^{\alpha \beta} \xi^{2} \eta X_{\alpha} u^{i} X_{\beta} u^{j} d z \\
& =\iint_{Q^{\prime} R}\left[\frac{1}{2}\left|u^{i}-\bar{u}(t)\right|^{2} \xi^{2} \eta_{t}-B_{i j}^{\alpha \beta} \xi^{2} \eta X_{\alpha} u^{i} X_{\beta} u^{j}\right] d z \\
& \quad-2 \iint_{Q^{\prime} R} a_{i j}^{\alpha \beta}\left(u^{i}-\bar{u}(t)\right) \xi \eta X_{\alpha} \xi X_{\beta} u^{j} d z
\end{aligned}
$$


Dong et al. Boundary Value Problems

(2019) 2019:166

Page 9 of 15

$$
+\iint_{Q^{\prime}{ }_{R}}\left[g_{i}\left(u^{i}-\bar{u}(t)\right) \xi^{2} \eta+2 \xi \eta\left(u^{i}-\bar{u}(t)\right) f_{i}^{\alpha} X_{\alpha} \xi+f_{i}^{\alpha} \xi^{2} \eta X_{\alpha} u^{i}\right] d z .
$$

Due to the skew-symmetry of $B_{i j}^{\alpha \beta}$,

$$
\iint_{Q^{\prime} R}\left(B_{i j}^{\alpha \beta}\right)_{R}\left(u^{i}-\bar{u}(t)\right) \xi \eta X_{\alpha} \xi X_{\beta} u^{j} d z=0 .
$$

By (H1), (3.10) and Young's inequality, we have

$$
\begin{aligned}
& \iint_{Q^{\prime_{R}}} a_{i j}^{\alpha \beta}\left(u^{i}-\bar{u}(t)\right) \xi \eta X_{\alpha} \xi X_{\beta} u^{j} d z \\
& =\iint_{Q_{R}^{\prime}} A_{i j}^{\alpha \beta}\left(u^{i}-\bar{u}(t)\right) \xi \eta X_{\alpha} \xi X_{\beta} u^{j} d z+\iint_{Q^{\prime} R} B_{i j}^{\alpha \beta}\left(u^{i}-\bar{u}(t)\right) \xi \eta X_{\alpha} \xi X_{\beta} u^{j} d z \\
& =\iint_{Q_{R}^{\prime}} A_{i j}^{\alpha \beta}\left(u^{i}-\bar{u}(t)\right) \xi \eta X_{\alpha} \xi X_{\beta} u^{j} d z \\
& \quad+\iint_{Q^{\prime}{ }_{R}}\left(B_{i j}^{\alpha \beta}-\left(B_{i j}^{\alpha \beta}\right)_{R}\right)\left(u^{i}-\bar{u}(t)\right) \xi \eta X_{\alpha} \xi X_{\beta} u^{j} d z \\
& \quad \leq \Lambda^{-1} \iint_{Q_{R}^{\prime}}|u-\bar{u}(t)||X \xi||X u| \xi \eta d z \\
& \quad+\iint_{Q^{\prime} R}\left|B_{i j}^{\alpha \beta}-\left(B_{i j}^{\alpha \beta}\right)_{R}\right||u-\bar{u}(t)||X \xi \| X u| \xi \eta d z \\
& \leq c_{\varepsilon} \iint_{Q_{R}^{\prime}}|u-\bar{u}(t)|^{2}|X \xi|^{2} \eta d z+2 \varepsilon \iint_{Q^{\prime}}|X u|^{2} \xi^{2} \eta d z \\
& \quad+c_{\varepsilon} \iint_{Q^{\prime}{ }_{R}}\left|B_{i j}^{\alpha \beta}-\left(B_{i j}^{\alpha \beta}\right)_{R}\right|^{2}|u-\bar{u}(t)|^{2}|X \xi|^{2} \eta d z .
\end{aligned}
$$

By Hölder's and Sobolev's inequalities, we have

$$
\begin{aligned}
& \iint_{Q_{R}^{\prime}}\left|B_{i j}^{\alpha \beta}-\left(B_{i j}^{\alpha \beta}\right)_{R}\right|^{2}|u-\bar{u}(t)|^{2} d z \\
& \quad \leq\left(\iint_{Q^{\prime}{ }_{R}}\left|B_{i j}^{\alpha \beta}-\left(B_{i j}^{\alpha \beta}\right)_{R}\right|^{Q} d z\right)^{\frac{2}{Q}}\left(\iint_{Q^{\prime} R}|u-\bar{u}(t)|^{\frac{2 Q}{Q-2}} d z\right)^{\frac{Q-2}{Q}} \\
& \quad \leq c\left|Q_{R}\right|^{\frac{2}{Q}} \cdot\|B\|_{\mathrm{BMO}}^{2}\left(\int_{I_{R}}\left(\int_{B_{R}}|X u|^{2} d x\right)^{\frac{Q}{Q-2}} d t\right)^{\frac{Q-2}{Q}} \\
& \quad \leq c\|B\|_{\mathrm{BMO}}^{2} R^{3}\left(\int_{I_{R}}\left(\int_{B_{R}}|X u|^{2} d x\right)^{2} d t\right)^{\frac{1}{2}} .
\end{aligned}
$$

Putting (3.12) into (3.11), we get

$$
\begin{aligned}
& \iint_{Q^{\prime} R} a_{i j}^{\alpha \beta}\left(u^{i}-\bar{u}(t)\right) \xi \eta X_{\alpha} \xi X_{\beta} u^{j} d z \\
& \quad \leq c_{\varepsilon} \iint_{Q^{\prime} R}|u-\bar{u}(t)|^{2}|X \xi|^{2} \eta d z+2 \varepsilon \iint_{Q^{\prime}}|X u|^{2} \xi^{2} \eta d z \\
& \quad+\frac{c_{\varepsilon}\|B\|_{\mathrm{BMO}}^{2} R^{3}}{(R-\rho)^{2}}\left(\int_{I_{R}}\left(\int_{B_{R}}|X u|^{2} d x\right)^{2} d t\right)^{\frac{1}{2}} .
\end{aligned}
$$


Using properties of $\xi(x), \eta(t)$ and (3.5),

$$
\begin{aligned}
& \iint_{Q^{\prime_{R}}} g_{i}\left(u^{i}-\bar{u}(t)\right) \xi^{2} \eta d z \\
& \leq \iint_{Q_{R}} g_{i}\left(u^{i}-\bar{u}(t)\right) d z \\
& \quad \leq c_{\varepsilon} \iint_{Q_{R}}|\tilde{g}|^{\tilde{q}} d z+\varepsilon R^{\frac{4}{Q}} \sup _{I_{R}}\left(\int_{B_{R}}|X u|^{2} d x\right)^{\frac{2}{Q}} \iint_{Q_{R}}|X u|^{2} d z \\
& \quad+c R^{\frac{Q+2-Q \gamma_{0}}{2}} \sup _{I_{R}}\left(\int_{B_{R}}|X u|^{2} d x\right)^{\frac{\gamma_{0}-1}{2}} \iint_{Q_{R}}|X u|^{2} d z .
\end{aligned}
$$

By (H2), Hölder's and Young's inequalities,

$$
\begin{aligned}
& \iint_{Q_{Q_{R}}}\left[2 \xi \eta\left(u^{i}-\bar{u}(t)\right) f_{i}^{\alpha} X_{\alpha} \xi+f_{i}^{\alpha} \xi^{2} \eta X_{\alpha} u^{i}\right] d z \\
& \leq 2 \iint_{Q_{R_{R}}}|u-\bar{u}(t)|\left|g_{i}^{\alpha}(z)\right||X \xi| \xi \eta d z+2 L \iint_{Q_{R_{R}}}|u-\bar{u}(t)||X u||X \xi| \xi \eta d z \\
& \quad+\iint_{Q_{Q_{R}}}\left|g_{i}^{\alpha}(z)\right||X u| \xi^{2} \eta d z+L \iint_{Q^{\prime}{ }_{R}}|X u|^{2} \xi^{2} \eta d z \\
& \leq 2 c_{\varepsilon} \iint_{Q^{\prime} R_{R}}|u-\bar{u}(t)|^{2}|X \xi|^{2} \eta d z+c_{\varepsilon} \iint_{Q^{\prime}{ }_{R}}|\tilde{\tilde{g}}|^{2} \xi^{2} \eta d z \\
& \quad+(2 \varepsilon+L) \iint_{Q_{Q_{R}}}|X u|^{2} \xi^{2} \eta d z .
\end{aligned}
$$

Inserting (3.13), (3.14), and (3.15) into (3.9), and by (H1), (3.3), (3.4), and Young's inequality, we get

$$
\begin{aligned}
\int_{B_{R}} \frac{1}{2}|u-\bar{u}(t)|^{2} \xi^{2} \eta d x+\Lambda \iint_{Q^{\prime}{ }_{R}}|X u|^{2} \xi^{2} \eta d z \\
\leq \iint_{Q^{\prime}{ }_{R}} \frac{1}{2}|u-\bar{u}(t)|^{2} \xi^{2} \eta_{t} d z+3 c_{\varepsilon} \iint_{Q^{\prime}{ }_{R}}|u-\bar{u}(t)|^{2}|X \xi|^{2} \eta d z \\
\quad+\frac{c_{\varepsilon}\|B\|_{\mathrm{BMO}}^{2} R^{3}}{(R-\rho)^{2}}\left(\int_{I_{R}}\left(\int_{B_{R}}|X u|^{2} d x\right)^{2} d t\right)^{\frac{1}{2}}+c_{\varepsilon} \iint_{Q_{R}}|\tilde{g}|^{\tilde{q}} d z \\
\quad+c_{\varepsilon} \iint_{Q_{R_{R}}}|\tilde{\tilde{g}}|^{2} \xi^{2} \eta d z+\theta_{1} \iint_{Q_{R}}|X u|^{2} d z,
\end{aligned}
$$

where $\theta_{1}=\varepsilon R^{\frac{4}{Q}} \sup _{I_{R}}\left(\int_{B_{R}}|X u|^{2} d x\right)^{\frac{2}{Q}}+c R^{\frac{Q+2-Q \gamma_{0}}{2}} \sup _{I_{R}}\left(\int_{B_{R}}|X u|^{2} d x\right)^{\frac{\gamma_{0}-1}{2}}+4 \varepsilon+L$. Employing properties of $\xi(x), \eta(t)$, (H1), and since $\frac{1}{R^{2}-\rho^{2}} \leq \frac{{ }_{C}}{(R-\rho)^{2}}$, we have

$$
\begin{aligned}
& \frac{1}{2} \sup _{I_{\rho}} \int_{B_{\rho}}|u-\bar{u}(t)|^{2} d x+\Lambda \iint_{Q_{\rho}}|X u|^{2} d z \\
& \quad \leq \frac{c}{(R-\rho)^{2}} \iint_{Q_{R}}|u-\bar{u}(t)|^{2} d z+\frac{c_{\varepsilon}\|B\|_{\mathrm{BMO}}^{2} R^{3}}{(R-\rho)^{2}}\left(\int_{I_{R}}\left(\int_{B_{R}}|X u|^{2} d x\right)^{2} d t\right)^{\frac{1}{2}} \\
& \quad+c_{\varepsilon} \iint_{Q_{R}}|\tilde{g}|^{\tilde{q}} d z+c_{\varepsilon} \iint_{Q_{R}}|\tilde{\tilde{g}}|^{2} d z+\theta_{1} \iint_{Q_{R}}|X u|^{2} d z .
\end{aligned}
$$


Dong et al. Boundary Value Problems

(2019) 2019:166

Page 11 of 15

Because $L<\Lambda$, by choosing $\varepsilon, R$ small enough we can get that $\theta_{1}<\Lambda$, so Lemma 2.2 yields

$$
\begin{aligned}
& \sup _{I_{\rho}} \int_{B_{\rho}}|u-\bar{u}(t)|^{2} d x+\iint_{Q_{\rho}}|X u|^{2} d z \\
& \leq \frac{c}{(R-\rho)^{2}} \iint_{Q_{R}}|u-\bar{u}(t)|^{2} d z+c \iint_{Q_{R}}|\tilde{g}|^{\tilde{q}} d z+c \iint_{Q_{R}}|\tilde{\tilde{g}}|^{2} d z \\
& \quad+\frac{c\|B\|_{\mathrm{BMO}}^{2} R^{3}}{(R-\rho)^{2}}\left(\sup _{I_{R}} \int_{B_{R}}|X u|^{2} d x\right)^{\frac{1}{2}}\left(\iint_{Q_{R}}|X u|^{2} d z\right)^{\frac{1}{2}} .
\end{aligned}
$$

By (3.1),

$$
\iint_{Q_{R}}|X u|^{2} d z \leq c \iint_{Q_{R}}|\tilde{g}|^{\tilde{q}} d z+c \iint_{Q_{R}}|\tilde{\tilde{g}}|^{2} d z
$$

Then

$$
\begin{aligned}
& \sup _{I_{\rho}} \int_{B_{\rho}}|u-\bar{u}(t)|^{2} d x+\iint_{Q_{\rho}}|X u|^{2} d z \\
& \leq \frac{c}{(R-\rho)^{2}} \iint_{Q_{R}}|u-\bar{u}(t)|^{2} d z+c \iint_{Q_{R}}|\tilde{g}|^{\tilde{q}} d z+c \iint_{Q_{R}}|\tilde{\tilde{g}}|^{2} d z \\
& \quad+\frac{c\|B\|_{\mathrm{BMO}}^{2} R^{3} \sup _{I_{R}} \int_{B_{R}}|X u|^{2} d x}{(R-\rho)^{2}}\left(\iint_{Q_{R}}\left(|\tilde{g}|^{\tilde{q}}+|\tilde{\tilde{g}}|^{2}\right) d z\right)^{\frac{1}{2}} \\
& \leq \frac{c}{(R-\rho)^{2}} \iint_{Q_{R}}|u-\bar{u}(t)|^{2} d z+c \iint_{Q_{R}}|\tilde{g}|^{\tilde{q}} d z+c \iint_{Q_{R}}|\tilde{\tilde{g}}|^{2} d z \\
& \quad+\frac{c\|B\|_{\mathrm{BMO}}^{2} R^{3} \sup _{I_{R}} \int_{B_{R}}|X u|^{2} d x}{(R-\rho)^{2}\left(\|\tilde{g}\|_{L^{\tilde{q}}}^{\tilde{q}}+\mid \tilde{\tilde{g}} \|_{L^{2}}^{2}\right)^{\frac{1}{2}}} \iint_{Q_{R}}\left(|\tilde{g}|^{\tilde{q}}+|\tilde{\tilde{g}}|^{2}\right) d z \\
& \leq \frac{c}{(R-\rho)^{2}} \iint_{Q_{R}}|u-\bar{u}(t)|^{2} d z+c\left(\frac{R^{3}}{(R-\rho)^{2}}+1\right) \iint_{Q_{R}}\left(|\tilde{g}|^{\tilde{q}}+|\tilde{\tilde{g}}|^{2}\right) d z .
\end{aligned}
$$

The proof is completed.

Lemma 3.3 Let $u \in W_{2}^{1,1}\left(\Omega_{T}, \mathbb{R}^{N}\right)$ be a weak solution of (1.1). Then there exists a positive constant $\varepsilon_{0}$ such that for any $p \in\left[2,2+\tilde{q} \varepsilon_{0}\right)$, we have $u \in L_{\mathrm{loc}}^{\frac{p \gamma}{2}}\left(Q_{T}\right), X u \in L_{\mathrm{loc}}^{p}\left(Q_{T}\right)$, and for any $Q_{2 R} \subset \subset Q_{T}$,

$$
\frac{1}{\left|Q_{R}\right|} \iint_{Q_{R}}|X u|^{p} d z \leq c\left[\left(\frac{1}{\left|Q_{2 R}\right|} \iint_{Q_{2 R}}|X u|^{2} d z\right)^{\frac{p}{2}}+\frac{1}{\left|Q_{2 R}\right|} \iint_{Q_{2 R}}\left(|\tilde{g}|^{\tilde{q}}+|\tilde{\tilde{g}}|^{2}\right)^{\frac{p}{2}} d z\right] .
$$

Proof By (3.7) and Sobolev's inequality,

$$
\begin{aligned}
& \sup _{I_{4 R / 5}}\left(\int_{B_{4 R / 5}}|u-\bar{u}(t)|^{2} d x\right)^{\frac{1}{2}} \\
& \quad \leq\left(\frac{c}{R^{2}} \iint_{Q_{R}}|u-\bar{u}(t)|^{2} d z\right)^{\frac{1}{2}}+c\left(\iint_{Q_{R}}\left(|\tilde{g}|^{\tilde{q}}+|\tilde{\tilde{g}}|^{2}\right) d z\right)^{\frac{1}{2}}
\end{aligned}
$$


Dong et al. Boundary Value Problems

(2019) 2019:166

Page 12 of 15

$$
\leq c\left(\iint_{Q_{R}}|X u|^{2} d z\right)^{\frac{1}{2}}+c\left(\iint_{Q_{R}}\left(|\tilde{g}|^{\tilde{q}}+|\tilde{\tilde{g}}|^{2}\right) d z\right)^{\frac{1}{2}} .
$$

By Hölder's and Sobolev's inequalities, it follows

$$
\begin{aligned}
& \int_{I_{4 R / 5}}\left(\int_{B_{4 R / / 5}}|u-\bar{u}(t)|^{2} d x\right)^{\frac{1}{2}} d t \\
& \quad \leq \int_{I_{R}}\left(\int_{B_{R}}|u-\bar{u}(t)|^{\tilde{q}} d x\right)^{\frac{1}{2 \tilde{q}}}\left(\int_{B_{R}}|u-\bar{u}(t)|^{\gamma} d x\right)^{\frac{1}{2 \gamma}} d t \\
& \quad \leq c R^{\frac{1}{\tilde{q}}} \int_{I_{R}}\left(\int_{B_{R}}|X u|^{\tilde{q}} d x\right)^{\frac{1}{2 \tilde{q}}}\left(\int_{B_{R}}|X u|^{2} d x\right)^{\frac{1}{4}} d t \\
& \quad \leq c R^{\frac{1}{\tilde{q}}}\left(\iint_{Q_{R}}|X u|^{\tilde{q}} d z\right)^{\frac{1}{2 \tilde{q}}}\left(\int_{I_{R}}\left(\int_{B_{R}}|X u|^{2} d x\right)^{\frac{1}{2} \frac{\tilde{q}}{2 \tilde{q}-1}} d t\right)^{\frac{2 \tilde{q}-1}{2 \tilde{q}}} \\
& \quad \leq c R^{\frac{3}{2}}\left(\iint_{Q_{R}}|X u|^{\tilde{q}} d z\right)^{\frac{1}{2 \tilde{q}}}\left(\iint_{Q_{R}}|X u|^{2} d z\right)^{\frac{1}{4}},
\end{aligned}
$$

where $\gamma=\frac{2(Q+2)}{Q}$. By (3.16) and (3.17),

$$
\begin{aligned}
& \iint_{Q_{4 R / 5}}|u-\bar{u}(t)|^{2} d z \\
& \quad=\int_{I_{4 R / 5}}\left(\int_{B_{4 R / 5}}|u-\bar{u}(t)|^{2} d x\right) d t \\
& \quad \leq \sup _{I_{4 R / 5}}\left(\int_{B_{4 R / 5}}|u-\bar{u}(t)|^{2} d x\right)^{\frac{1}{2}} \cdot\left(\int_{I_{4 R / 5}}\left(\int_{B_{4 R / 5}}|u-\bar{u}(t)|^{2} d x\right)^{\frac{1}{2}} d t\right) \\
& \quad \leq c R^{\frac{3}{2}}\left(\iint_{Q_{R}}|X u|^{\tilde{q}} d z\right)^{\frac{1}{2 \tilde{q}}}\left(\iint_{Q_{R}}|X u|^{2} d z\right)^{\frac{3}{4}} \\
& \quad+c R^{\frac{3}{2}}\left(\iint_{Q_{R}}|X u|^{\tilde{q}} d z\right)^{\frac{1}{2 \tilde{q}}}\left(\iint_{Q_{R}}|X u|^{2} d z\right)^{\frac{1}{4}}\left(\iint_{Q_{R}}\left(|\tilde{g}|^{\tilde{q}}+|\tilde{\tilde{g}}|^{2}\right) d z\right)^{\frac{1}{2}} \\
& \quad \equiv I_{1}+I_{2} .
\end{aligned}
$$

By Young's inequality,

$$
\begin{aligned}
I_{1} & \leq c_{\varepsilon}\left(\iint_{Q_{R}}|X u|^{\tilde{q}} d z\right)^{\frac{2}{\tilde{q}}}+\varepsilon R^{2} \iint_{Q_{R}}|X u|^{2} d z \\
I_{2} & \leq \varepsilon R\left(\iint_{Q_{R}}|X u|^{\tilde{q}} d z\right)^{\frac{1}{\tilde{q}}}\left(\iint_{Q_{R}}|X u|^{2} d z\right)^{\frac{1}{2}}+c_{\varepsilon} R^{2} \iint_{Q_{R}}\left(|\tilde{g}|^{\tilde{q}}+|\tilde{\tilde{g}}|^{2}\right) d z \\
& \leq c_{\varepsilon}\left(\iint_{Q_{R}}|X u|^{\tilde{q}} d z\right)^{\frac{2}{\tilde{q}}}+\varepsilon R^{2} \iint_{Q_{R}}|X u|^{2} d z+c_{\varepsilon} R^{2} \iint_{Q_{R}}\left(|\tilde{g}|^{\tilde{q}}+|\tilde{\tilde{g}}|^{2}\right) d z .
\end{aligned}
$$

Inserting the estimates of $I_{1}$ and $I_{2}$ into (3.18), we get

$$
\iint_{Q_{4 R / 5}}|u-\bar{u}(t)|^{2} d z
$$




$$
\leq c_{\varepsilon}\left(\iint_{Q_{R}}|X u|^{\tilde{q}} d z\right)^{\frac{2}{\tilde{q}}}+\varepsilon R^{2} \iint_{Q_{R}}|X u|^{2} d z+c_{\varepsilon} R^{2} \iint_{Q_{R}}\left(|\tilde{g}|^{\tilde{q}}+|\tilde{\tilde{g}}|^{2}\right) d z .
$$

By (3.7) and (3.19),

$$
\begin{aligned}
& \frac{1}{\left|Q_{3 R / 4}\right|} \iint_{Q_{3 R / 4}}|X u|^{2} d z \\
& \leq \frac{c}{R^{2}} \frac{1}{\left|Q_{3 R / 4}\right|} \iint_{Q_{4 R / 5}}|u-\bar{u}(t)|^{2} d z+\frac{c}{\left|Q_{3 R / 4}\right|} \iint_{Q_{4 R / 5}}\left(|\tilde{g}|^{\tilde{q}}+|\tilde{\tilde{g}}|^{2}\right) d z \\
& \leq \frac{c_{\varepsilon}\left|Q_{R}\right|^{\frac{2}{\tilde{q}}}}{\left|Q_{3 R / 4}\right| R^{2}}\left(\frac{1}{\left|Q_{R}\right|} \iint_{Q_{R}}|X u|^{\tilde{q}} d z\right)^{\frac{2}{\tilde{q}}}+\frac{\varepsilon}{\left|Q_{3 R / 4}\right|} \iint_{Q_{R}}|X u|^{2} d z \\
& \quad+\frac{c_{\varepsilon}}{\left|Q_{3 R / 4}\right|} \iint_{Q_{R}}\left(|\tilde{g}|^{\tilde{q}}+|\tilde{\tilde{g}}|^{2}\right) d z \\
& \leq c_{\varepsilon}\left(\frac{1}{\left|Q_{R}\right|} \iint_{Q_{R}}|X u|^{\tilde{q}} d z\right)^{\frac{2}{\tilde{q}}}+\frac{\varepsilon}{\left|Q_{R}\right|} \iint_{Q_{R}}|X u|^{2} d z \\
& \quad+\frac{c_{\varepsilon}}{\left|Q_{R}\right|} \iint_{Q_{R}}\left(|\tilde{g}|^{\tilde{q}}+|\tilde{\tilde{g}}|^{2}\right) d z .
\end{aligned}
$$

Let $\hat{g}=|X u|^{\tilde{q}}\left(\hat{q}=\frac{2}{\tilde{q}}=\frac{Q+4}{Q+2}>1\right), \hat{f}=\left(|\tilde{g}|^{\tilde{q}}+|\tilde{\tilde{g}}|^{2}\right)^{\tilde{q}}$, then the above can be written as

$$
\frac{1}{\left|Q_{3 R / 4}\right|} \iint_{Q_{3 R / 4}} \hat{g}^{\hat{q}} d z \leq c\left[\left(\frac{1}{\left|Q_{R}\right|} \iint_{Q_{R}} \hat{g} d z\right)^{\hat{q}}+\frac{1}{\left|Q_{R}\right|} \iint_{Q_{R}} \hat{f}^{\hat{q}} d z\right]+\frac{\varepsilon}{\left|Q_{R}\right|} \iint_{Q_{R}} \hat{g}^{\hat{q}} d z .
$$

By Lemma 2.3, we know that there exists a positive constant $\varepsilon_{0}$ such that for any $\hat{p} \in$ $\left[\hat{q}, \hat{q}+\varepsilon_{0}\right)$,

$$
\begin{aligned}
& \left(\frac{1}{\left|Q_{R}\right|} \iint_{Q_{R}}|X u|^{\hat{\tilde{q}} \tilde{q}} d z\right)^{\frac{1}{\hat{p}}} \\
& \quad \leq c\left[\left(\frac{1}{\left|Q_{2 R}\right|} \iint_{Q_{2 R}}|X u|^{2} d z\right)^{\frac{\tilde{q}}{2}}+\left(\frac{1}{\left|Q_{2 R}\right|} \iint_{Q_{2 R}}\left(|\tilde{g}|^{\tilde{q}}+|\tilde{\tilde{g}}|^{2}\right)^{\frac{\hat{p} \tilde{q}}{2}} d z\right)^{\frac{1}{\hat{p}}}\right] .
\end{aligned}
$$

Letting $p=\hat{p} \tilde{q} \in\left[2,2+\tilde{q} \varepsilon_{0}\right)$, we finish the proof.

Proof of Theorem 1.1 By (3.1), Lemma 3.3, and Hölder's inequality, we have

$$
\begin{aligned}
& \iint_{Q_{R}}|X u|^{p} d z \\
& \quad \leq c\left|Q_{R}\right|\left[\left(\frac{1}{\left|Q_{2 R}\right|} \iint_{Q_{2 R}}\left(|\tilde{g}|^{\tilde{q}}+|\tilde{\tilde{g}}|^{2}\right) d z\right)^{\frac{p}{2}}+\frac{1}{\left|Q_{2 R}\right|} \iint_{Q_{2 R}}\left(|\tilde{g}|^{\tilde{q}}+|\tilde{\tilde{g}}|^{2}\right)^{\frac{p}{2}} d z\right] \\
& \quad \leq c \iint_{Q_{2 R}}\left(|\tilde{g}|^{\tilde{q}}+|\tilde{\tilde{g}}|^{2}\right)^{\frac{p}{2}} d z \leq c \iint_{Q_{2 R}}\left(|\tilde{g}|^{p q_{0}}+|\tilde{\tilde{g}}|^{p}\right) d z \\
& \quad \leq c\left(\|\tilde{g}\|_{L^{p q_{0}}}^{p q_{0}}+\|\tilde{\tilde{g}}\|_{L^{p}}^{p}\right) .
\end{aligned}
$$

The proof is completed. 


\section{Funding}

This work is supported by the National Natural Science Foundation of China (11701162); National Science Foundation of Shandong Province of China (ZR2019MA067); Research Fund for the Doctoral Program of Hubei University of Economics (XJ16BS28); Guangxi Natural Science Foundation (2017GXNSFBA198130).

\section{Availability of data and materials}

Not applicable.

\section{Ethics approval and consent to participate}

Not applicable.

\section{Competing interests}

The authors declare that they have no competing interests.

\section{Consent for publication}

Not applicable.

\section{Authors' contributions}

All authors read and approved the final manuscript.

\section{Author details}

'Department of Applied Mathematics, Hubei University Of Economics, Wuhan, China. ${ }^{2}$ School of Mathematical Sciences, Qufu Normal University, Qufu, China. ${ }^{3}$ School of Mathematics and Computing Science, Guilin University of Electronic Technology, Guilin, China

\section{Publisher's Note}

Springer Nature remains neutral with regard to jurisdictional claims in published maps and institutional affiliations.

Received: 12 July 2019 Accepted: 14 October 2019 Published online: 21 October 2019

\section{References}

1. Campanato, S.: LP regularity for weak solutions of parabolic systems. Ann. Sc. Norm. Super. Pisa, Cl. Sci. 7, 65-85 (1980)

2. Giaquinta, M., Struwe, M.: On the partial regularity of weak solutions on nonlinear parabolic systems. Math. Z. 179, 437-451 (1982)

3. Daněček, J., Viszus, E.: A note on regularity for nonlinear elliptic systems. Arch. Math. 36(3), 229-237 (2000)

4. Dong, H., Kim, D.: Global regularity of weak solutions to quasilinear elliptic and parabolic equations with controlled growth. Commun. Partial Differ. Equ. 36(10-12), 1750-1777 (2011)

5. Giaquinta, M., Modica, G.: Regularity results for some classes of higher order non linear elliptic systems. J. Reine Angew. Math. 311-312, 145-169 (1979)

6. Li, L., Pipher, J.: Boundary behavior of solutions of elliptic operators in divergence form with a BMO anti-symmetric part. Commun. Partial Differ. Equ. 44(2), 156-204 (2019)

7. Meier, M.: Liouville theorems for nondiagonal elliptic systems in arbitrary dimensions. Math. Z. 176, 123-133 (1981)

8. Phan, T.: Regularity gradient estimates for weak solutions of singular quasi-linear parabolic equations. J. Differ. Equ. 263(12), 8329-8361 (2017)

9. Wiegner, M.: Regularity theorems for nondiagonal elliptic systems. Ark. Mat. 20, 1-13 (1982)

10. Földes, J., Phan, T.: On higher integrability estimates for elliptic equations with singular coefficients (2018) arXiv:1804.03180

11. Hörmander, L.: Hypoelliptic second order differential equations. Acta Math. 119, 147-171 (1967)

12. Lu, G.: Weighted Poincaré and Sobolev inequalities for vector fields satisfying Hörmander's condition and applications. Rev. Mat. Iberoam. 8(3), 367-439 (1992)

13. Gianazza, U.: Regularity for nonlinear equations involving square Hörmander operators. Nonlinear Anal. 23(1), 49-73 (1994)

14. Di Fazio, G., Fanciullo, M.S.: Gradient estimates for elliptic systems in Carnot-Carathéodory spaces. Comment. Math. Univ. Carol. 43(4), 605-618 (2002)

15. Dong, Y., Niu, P.: Estimates in Morrey spaces and Hölder continuity for weak solutions to degenerate elliptic systems. Manuscr. Math. 138(3-4), 419-437 (2012)

16. Dong, Y:: Hölder regularity for weak solutions to divergence form degenerate quasilinear parabolic systems. J. Math Anal. Appl. 410(1), 375-390 (2014)

17. Dong, Y., Niu, P.: Regularity for weak solutions to nondiagonal quasilinear degenerate elliptic systems. J. Funct. Anal. 270(7), 2383-2414 (2016)

18. Dong, Y., Li, D.: Regularity for weak solutions to nondiagonal quasilinear degenerate parabolic systems with controllable growth conditions. N.Y. J. Math. 24, 53-81 (2018)

19. Du, G., Li, F.: Global higher integrability of solutions to subelliptic double obstacle problems. J. Appl. Anal. Comput. 8(3), 1021-1032 (2018)

20. Du, G., Li, F.: Interior regularity of obstacle problems for nonlinear subelliptic systems with VMO coefficients. J. Inequal. Appl. 201853 (2018)

21. Wang, J., Manfredi, J.: Partial Hölder continuity for nonlinear sub-elliptic systems with VMO-coefficients in the Heisenberg group. Adv. Nonlinear Anal. 7(1), 97-116 (2018)

22. Nagel, A., Stein, E.M., Wainger, S.: Balls and metrics defined by vector fields. I: basic properties. Acta Math. 155, 103-147 (1985)

23. Hajłasz, P., Koskela, P.: Sobolev met Poincaré. Mem. Am. Math. Soc. 688, 101 (2000) 
24. Xu, C., Zuily, C.: Higher interior regularity for quasilinear subelliptic systems. Calc. Var. Partial Differ. Equ. 5(4), 323-343 (1997)

25. Chen, Y., Wu, L.: Second Order Elliptic Equations and Elliptic Systems. American Mathematical Society, Providence (1998)

26. Zatorska-Goldstein, A.: Very weak solutions of nonlinear subelliptic equations. Ann. Acad. Sci. Fenn., Math. 30(2), 407-436 (2005)

Submit your manuscript to a SpringerOpen ${ }^{\mathcal{O}}$ journal and benefit from:

- Convenient online submission

Rigorous peer review

- Open access: articles freely available online

- High visibility within the field

- Retaining the copyright to your article

Submit your next manuscript at $\boldsymbol{\nabla}$ springeropen.com 